Sarah Torres Cajo

\title{
Zwischen Strukturierung, Wissensmanagement und Argumentation im Gespräch - Interaktionale Verwendungsweisen der Modalpartikeln halt und eben im gesprochenen Deutsch
}

\begin{abstract}
Im vorliegenden Beitrag werden auf der Grundlage authentischer Alltagsinteraktionen die Funktionsspektren der Modalpartikeln eben und halt beschrieben. Es wird dargelegt, dass die Partikeln keinesfalls synonym verwendet werden, sondern distinktive Verwendungen aufweisen. Für halt zeigen sich die drei prominenten Verwendungsweisen i) der Kohärenzherstellung, ii) der Verweis auf gemeinsames Wissen und iii) der Einsatz zur informationsstrukturellen (Beitrags-)Gliederung. Eben wird ebenfalls häufig zur Kohärenzherstellung eingesetzt, und trägt darüber hinaus besonders häufig als strategische Ressource zur rhetorischen Verstärkung einer Argumentation oder Schlussfolgerung bei. Abschließend werden die in der Literatur oft behauptete Ersetzbarkeit von halt durch eben und umgekehrt sowie die häufig zugeschriebene Konnotation von Unabänderlichkeit und Sprecherresignation diskutiert.

Based on authentic everyday interaction, this article describes the functional range of the German modal particles halt and eben. It will be shown that the particles are by no means used synonymously, as has frequently been postulated in studies of modal particles, but have distinctive uses in interaction. Thus, for halt there are three main functions: i) marking coherence, ii) referring to common knowledge, and iii) structuring information. Although eben can also be used to create coherence, speakers frequently use this modal particle as a strategic resource in argumentative discourses to increase the validity of the argument. Finally, on the basis of the previous analyses I will discuss the frequently claimed interchangeability of halt and eben as well as the commonly assumed connotation of inalterability and resignation on the part of the speaker.
\end{abstract}

\section{Einleitung ${ }^{1,2}$}

Spätestens seit der Arbeit von Thurmair (1989) stehen Modalpartikeln ${ }^{3}$ (MPn) im Fokus linguistischer Studien. Während Thurmair (1989) Modalpartikeln noch nicht als eigene Wortart, sondern als Funktionsvariante einer Gesamtklasse von Partikeln sieht, stufen die meisten Grammatiken Modalpartikeln heute als eigenständige Wortart ein (u. a. Eisenberg 1999; Helbig 1999; Helbig/Buscha 2001; Hentschel/Weydt 2003; Imo 2016). ${ }^{4}$ Imo (2016, S. 108) postuliert eine höflichkeitserzeugende Wirkung von Modalpartikeln, weshalb sie „,in der interaktionalen Sprache besonders oft [vorkommen]“ und fasst folgende Merk-

1 Dieser Beitrag ist im Rahmen des von der Leibniz-Gemeinschaft geförderten Projekts „Lexik des gesprochenen Deutsch“ entstanden (Förderlinie: „Innovative Vorhaben“), das am Leibniz-Institut für Deutsche Sprache (Mannheim) von September 2016 bis Oktober 2019 durchgeführt wurde. Weitere Informationen finden sich online unter www1.ids-mannheim.de/lexik/lexik-des-gesprochenen-deutsch. html.

2 Ich danke Katja Arens, Henrike Helmer sowie Stefan Engelberg ganz herzlich für wertvolle Kommentare, Anmerkungen und Diskussionen zu diesem Beitrag.

3 Ohne näher auf die terminologische Diskussion eingehen zu wollen, sei an dieser Stelle angemerkt, dass neben dem hier verwendeten Terminus Modalpartikel auch die Bezeichnungen Abtönungspartikel bzw. (seltener) Diskurspartikel möglich sind (für einen Überblick vgl. z. B. Blühdorn 2019).

4 Schoonjans (2013) diskutiert die Schwierigkeiten beim Versuch einer Kategoriendefinition der Modalpartikeln. 
male zusammen: i) Modalpartikeln sind nicht vorfeldfähig. ${ }^{5}$ ii) Sie können an bestimmte Satztypen gebunden sein (z. B. denn in Fragesätzen, halt in Aussagesätzen usw.). iii) Sie können ohne semantischen Verlust weggelassen werden und iv) ihre primäre Funktion besteht darin, einen dialogischen Bezug herzustellen; sie sind ,sozusagen Kurzformen für einen Sprecherkommentar“ (ebd., S. 108 f.). Zifonun/Hoffmann/Strecker (1997, S. 59) fassen für die Funktion von Modalpartikeln (dort: Abtönungspartikeln) zusammen, dass sie ,zur Einpassung der kommunikativen Minimaleinheit in den jeweiligen Handlungszusammenhang [beitragen], indem sie auf den Erwartungen und Einstellungen des Sprechers und der Adressaten operieren". MPn übernehmen also eine konnektive Funktion zwischen fokaler Äußerung und pragmatischem bzw. interaktionalem Prätext (vgl. auch Diewald 2006; Diewald/Kresić 2010). Diewald (2006, S. 415 f.) stellt fest: „,[T]here is general agreement on the relational, respectively indexical nature of MPs." Daher liegt eine Schwierigkeit bei der Beschreibung einzelner MPn oftmals darin, ihren spezifischen Referenzpunkt auszumachen (ebd., S. 416).

Mit der Referenz auf einen pragmatischen Prätext geht zugleich ein Verweis auf gemeinsames Wissen der Interagierenden einher, MPn operieren also auf intersubjektiver Ebene als Marker für (nicht notwendigerweise verbalisierten) common ground (vgl. Fischer 2007, S. 51). ${ }^{6}$ Deppermann (2009, S. 23, Hervorhebung i. O.) betont die Bedeutung von Modalpartikeln für Prozesse der Verstehensdokumentation in der Interaktion:

Im Deutschen [...] ist das System der Modalpartikeln und Diskursmarker besonders darauf spezialisiert, rekurrente Verstehenskonstellationen zu indizieren (wie z.B. die Unterstellung von geteiltem Wissen und Erwartungskongruenz durch ja, eben, halt, gell, natürlich, Erwartungsdiskrepanz bzw. Überraschung durch doch, auch, neu gewonnenes bzw. revidiertes Verständnis durch aha a(c)hso, Interpretationsunsicherheit durch vielleicht, wohl etc. [...]).

Der vorliegende Beitrag widmet sich den Modalpartikeln halt und eben. In den meisten Abhandlungen werden diese gemeinsam betrachtet und dabei oftmals als frei miteinander austauschbar oder sogar synonym bezeichnet (z. B. Weydt 1969; Hentschel 1986; Kwon 2005; Brünjes 2014). Frühe Arbeiten gehen von dialektalen (semantisch gleichen) Varianten aus und stellen eine klare regionale Trennung von halt im Süden und eben im Norden Deutschlands fest (vgl. Eichhoff 1978). Fragebogenumfragen haben zudem regional unterschiedliche Konnotationen aufgezeigt, wobei eben als ,eher kalt, klar, stark, aktiv, selbstbewusst und egoistisch“ (Hentschel 1986, S. 185) empfunden würde, während halt als warme und herzliche Variante wahrgenommen würde (ebd., S. 193: vgl. auch Helbig/ Helbig 1995). Spätere Untersuchungen zeigen eine landesübergreifende Koexistenz beider MPn und vermuten eine Etablierung in den 1980er Jahren (vgl. Dittmar 1997, 2000). Dittmar (1997, S. 299) begründet den Abgrenzungsprozess mit dem kommunikativen „Mehrwert“ der Partikel halt gegenüber eben im Sinne einer stilistisch-positiven Alltagsrhetorik, da halt freundlicher und weicher wirkt als eben.

Thurmair (1989) geht davon aus, dass beide Modalpartikeln grundlegend eine ähnliche Bedeutung haben und die Gültigkeit einer Aussage betreffen, die unterschiedlich stark beeinflusst werden kann, weshalb der tatsächliche Bedeutungsunterschied im Grad der Wahrscheinlichkeit liegt, der durch die MP modalisiert wird. Beide MPn sind rückwärts-

5 Allerdings weist Imo (2008) anhand der Modalpartikel halt nach, dass es durchaus Stellungsvarianten im Vor-Vorfeld, Vorfeld und Nachfeld geben kann.

6 Für einen Überblick zum Zusammenhang von Modalpartikeln und geteiltem Wissen siehe Reineke (2016, S. 81). 
gerichtet, beziehen sich also auf eine Vorgängeräußerung und weisen dabei oft eine kausale Relation auf (Müller 2018; vgl. auch Weydt 1969). Blühdorn (2019, S. 298) betont die epistemische Komponente von halt und eben und konstatiert, dass diese MPn dann zum Einsatz kommen, wenn ,nach fehlenden Argumenten, Erklärungen oder Schlussfolgerungen gesucht wird, die aktuell benötigt werden, mit den bisher verwendeten Werkzeugen aber noch nicht gefunden werden konnten“" (vgl. auch Diewald 2013; Müller 2018). Damit tragen beide Modalpartikeln zur Schließung lokaler Kohärenzlücken bei ,und beide zeigen an, dass der Sprecher zu diesem Zweck einen Wechsel der zugrundegelegten Wissensbasis vollzieht" (Blühdorn 2019, S. 299). Darüber hinaus werden beiden Partikeln oftmals die Aspekte „Unabänderlichkeit“" und „Resignation des Sprechers bezüglich des Sachverhalts" unterstellt (Hentschel 1986; Helbig 1988). Allerdings weist bereits Ickler (1994, S. 380) darauf hin, dass in vielen Fällen der generelle Kontext der Proposition in die Partikelbedeutung hineinprojiziert wird, wenn Beispielsätze wie „Da kann man halt nichts machen“ oder „Das ist eben so“ zur Illustration der Resignationskonnotation herangezogen werden (für eine Diskussion dieser Aspekte vgl. Kap. 5).

Obwohl viele Autor/innen halt und eben gemeinsam behandeln und von einer geteilten Bedeutungsbasis ausgehen, wird doch an einigen Stellen auf Unterschiede eingegangen:

- eben: Thurmair (1989, S. 120 f.) ordnet eben die Merkmale „Evidenz“ und „Konnex“ zu, geht also von einer (kausalen) Verbindung zwischen der eben-Äußerung und der Vorgängeräußerung aus, welche durch die MP als einzig mögliche Begründung bzw. Erklärung markiert wird. Daher kommt sie häufig in allgemeingültigen Aussagen, Rechtfertigungen und Entschuldigungen vor (vgl. auch Brauße 1986; Rost-Roth 1998). Blühdorn (2019, S. 300) weist darauf hin, dass Sprecher/innen mit der Nutzung von eben die Orientierung am aktuellen Wissen des Adressaten/der Adressatin vorübergehend aufgeben, um auf eine subjektive, bereits vorher genutzte Wissensbasis zurückzugreifen.

- halt: Eine Äußerung wird mit halt als plausible Erklärung oder Begründung markiert, zu der es durchaus alternative Erklärungen geben kann; es erhält also die Merkmale „plausibel“ und „Konnex“ (Thurmair 1989, S. 123 f.). Im Gegensatz zu eben kann halt als Mittel eingesetzt werden, eine Aussage zu bekräftigen, ohne sie kategorisch oder „rechthaberisch“ wirken zu lassen. Äußerungen mit halt scheinen „abgeschwächter zu sein, weniger apodiktisch und verbindlicher“ (ebd., S. 125). Ebenfalls anders als mit eben greifen Sprecher/innen mit halt nicht auf individuelles, sondern auf unabhängiges, im gegebenen Kontext allgemein zugängliches Wissen zurück, das aller Wahrscheinlichkeit nach für den Rezipienten/die Rezipientin verfügbar ist (Blühdorn 2019, S. 302).

Trotz dieser Vielzahl von Erwähnungen und Merkmalsauflistungen liegen nach meinen Recherchen derzeit noch keine ausführlichen interaktionslinguistischen Arbeiten vor, die sich mit dem spezifischen Bedeutungs- und Funktionsspektrum von halt und eben beschäftigen. Im Gegensatz zu vielen anderen individuell beschriebenen (Modal-)Partikeln ${ }^{7}$ wurden halt und eben bisher hauptsächlich innerhalb größerer Abhandlungen bezüglich Modalpartikeln allgemein betrachtet oder unter anderen Gesichtspunkten fokussiert (vgl. z.B. Imo 2008, der Stellungsvarianten von Modalpartikeln beispielhaft anhand von halt

Vgl. beispielsweise Deppermann (2009) zu denn in Fragen, Spreckels (2009) zu einfach, Diewald/ Fischer (1998) zu aber, auch, doch und ja, Reineke (2016) zu ja. 
analysiert). Das Ziel des vorliegenden Beitrags ist es daher, die MPn halt und eben ganzheitlich in ihrer Verwendung in interaktionalen Kontexten anhand von authentischen Gesprächsdaten zu betrachten und dabei das jeweilige Funktions- und Bedeutungsspektrum aufzuzeigen. Die Analysen erfolgen im Rahmen eines interaktionslinguistischen Ansatzes (Selting/Couper-Kuhlen 2000), wobei die Partikeln innerhalb ihrer kontextuellen Umgebung betrachtet werden. Deppermann (2009, S. 23) weist darauf hin, dass Modalpartikeln im Allgemeinen ,semantisch ,leichte ${ }^{6}$ Kontextualisierungsanweisungen sind, die hochgradig kontextfrei einsetzbar sein müssen und sich andererseits auf hochgradig kontextspezifische Konstellationen und Sachverhalte beziehen", was eine Funktionsbestimmung oftmals schwierig macht. „Die Vielfalt der pragmatischen Funktionen der Äußerungen, in denen sie vorkommen [und] die Schwierigkeit, die funktionale Rolle der Partikel als solche zu isolieren" stellen grundlegende Herausforderungen bei der Analyse von Partikeln dar (ebd., S. 49).

Im vorliegenden Beitrag wird die These vertreten, dass es zwar Funktionsüberschneidungen zwischen den MPn halt und eben gibt, sich die Partikeln jedoch in ihren spezifischen Verwendungspraktiken voneinander unterscheiden, weshalb eine individuelle Betrachtung notwendig ist. Im Folgenden werde ich daher die Verwendungen von halt und eben zunächst unabhängig voneinander diskutieren, um im Anschluss in einem zusammenfassenden Kapitel spezifisch auf Gemeinsamkeiten und Unterschiede einzugehen.

\section{Datengrundlage und Vorgehen}

Die vorliegende Studie beruht auf Belegen aus dem Forschungs- und Lehrkorpus Gesprochenes Deutsch (FOLK), welches über die Datenbank für Gesprochenes Deutsch ${ }^{8}$ online verfügbar ist. FOLK enthält aktuell 259 Gesprächsereignisse mit über 2 Millionen transkribierten und annotierten Token in über 279 Stunden Audio- (und Video-)Aufnahmen ${ }^{9}$ aus informellen, institutionellen und öffentlichen Interaktionskontexten. Aus dem Korpus wurden Zufallsstichproben von jeweils 300 Vorkommen gezogen, von denen jeweils 100 Fälle auf spezifische Parameter kodiert wurden. Für halt handelt es sich bei allen Vorkommen um die Modalpartikel, während eben in 79 Fällen als Partikel, in 20 als Adverb und einmal als Adjektiv auftritt. Von diesen 79 Partikelvorkommen entfallen zwölf auf die Diskurspartikel, sodass sich eine eben-Gesamtstichprobe von 67 Modalpartikelvorkommen ergibt. ${ }^{10}$ Das Kodierschema wurde mithilfe qualitativer Einzelfallanalysen entwickelt und beinhaltet Informationen bezüglich Sprechhandlungen im Prä- und Postkontext, sequenzieller Realisierung, kombinatorischer Elemente, prosodischer Realisierung und interaktiver Funktion.

\section{Verwendungsweisen der MP halt im gesprochenen Deutsch}

Für die MP halt lassen sich in der untersuchten Stichprobe drei mehr oder weniger distinktive Funktionen ausmachen, die sich im Einzelnen überschneiden können und daher nicht als trennscharf anzusehen sind. Die beiden in der Literatur beschriebenen Funktionen von Kohärenzherstellung sowie vom Verweis auf gemeinsames Wissen sind ebenso

\footnotetext{
Link: http://dgd.ids-mannheim.de.

Stand der Version 2.10: Juli 2018.

10 In diesem Beitrag wird nur die Modalpartikelverwendung von eben betrachtet. Für eine Analyse der Formen und Funktionen von eben als Diskurspartikel vgl. Betz/Deppermann (2018).
} 
in den vorliegenden Daten prominent vertreten. Zudem wird halt rekurrent zur inhaltlichen Strukturierung von Gesprächsbeiträgen genutzt, wobei es sowohl zentrale als auch Nebeninformationen markieren kann. Diese Funktionen werden im Folgenden detailliert diskutiert.

\section{1 halt zur Kohärenzherstellung}

Eine prominente Verwendungsweise - und oftmals als Teil der Grundbedeutung von halt postuliert - ist die Kohärenzherstellung. Blühdorn (2019, S. 301) spricht von der „Schließung einer Kohärenzlücke [...], die sich in der aktuellen (Inter-)Aktion aufgetan hat". Entsprechend geht es bei ihm vor allem um inhaltliche Kohärenz, also die Lieferung von Informationen zur inhaltlichen Verknüpfung. In unseren Daten findet sich zusätzlich zu dieser Funktion auch die Verknüpfung auf sequenzieller Ebene. ${ }^{11}$

Zunächst ein Beleg für die inhaltliche Verknüpfung: Im folgenden Beispiel unterhalten sich vier Mitbewohnerinnen, die erst seit kurzen zusammenwohnen, über das Anbringen von Namensschildern an der Klingel bzw. am Briefkasten des Hauses. Die Sprecherin NH hatte in der Vergangenheit ein handgeschriebenes Schild angebracht, was bei der Vermieterin auf Unwillen gestoßen war. In der folgenden Sequenz rechtfertigt NH dieses Verhalten.

Beispiel 1: inhaltliche Kohärenzherstellung [FOLK_E_00055_SE_01_T_05, c37] ${ }^{12}$

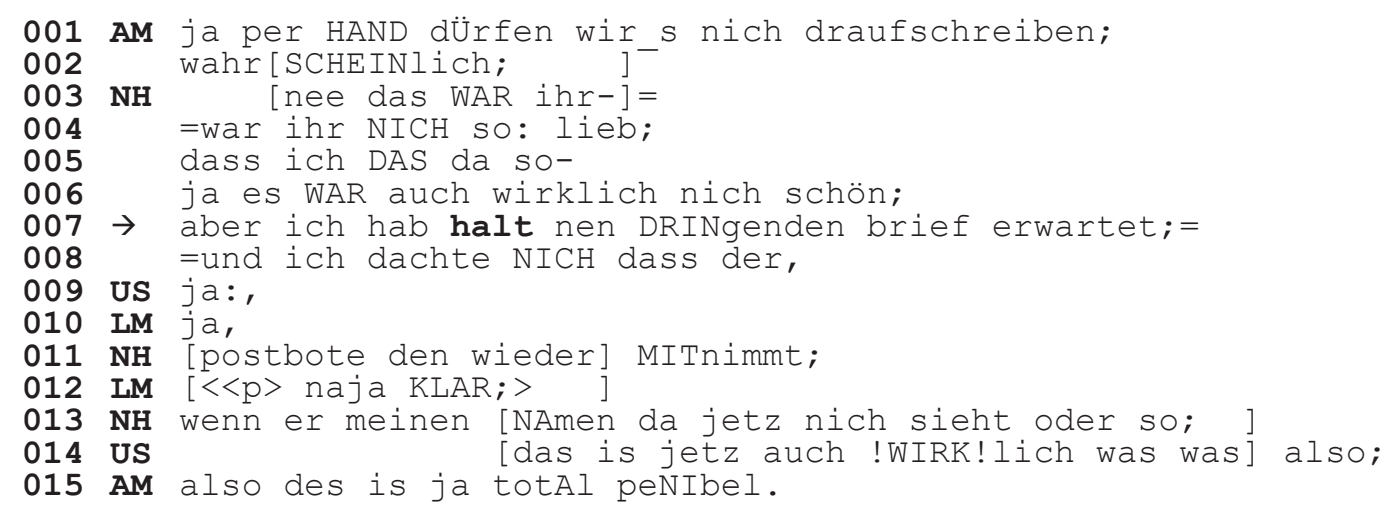

AM vermutet zu Beginn des Ausschnitts, dass Namensschilder nicht handgeschrieben angebracht werden dürfen. NH bestätigt die Vermutung mit der Negationspartikel nee und steigt in eine kurze Rekonstruktion der Reaktion ihrer Vermieterin ein (Z. $003 \mathrm{ff}$.). Sie bricht ihre Erzählung ab und bewertet stattdessen das Resultat ihrer Handlung als ,wirklich nich schön“ (Z. 006). Indem sie selbst einräumt, dass das Resultat nicht optimal war, macht sie prospektiv eine Erklärung relevant, um ihre Handlung zu rechtfertigen. ${ }^{13}$ Die

11 Eine klare Distinktion zwischen inhaltlicher und sequenzieller Ebene ist selbstverständlich nicht immer so trennscharf möglich, wie hier dargestellt. Jedoch lassen sich mithilfe detaillierter turn-by-turn-Analysen Unterschiede feststellen, die eine Interpretation in die eine oder die andere Richtung wahrscheinlicher machen.

12 Alle Belege wurden nach den in Selting et al. (2009) beschriebenen Transkriptionskonventionen als Basistranskripte transkribiert.

13 Die negative Selbstbewertung stellt zudem eine face-bedrohende Situation für NH her, durch die ein rechtfertigungsrelevanter Raum eröffnet wird (zu face vgl. Goffman 1967 sowie für face-threatening acts (FTAs) Brown/Levinson 1987). 
adversative Konjunktion aber leitet die Rechtfertigung ein und deutet bereits auf ein gewichtigeres Gegenargument hin (vgl. auch Günthner 2015): ,aber ich hab halt nen DRINgenden brief erwartet" (Z. 007). Die Aussage knüpft an das allgemeine Hintergrundwissen aller Teilnehmerinnen an, dass Briefe nur dann beim Empfänger ankommen, wenn der Name am Briefkasten ausgewiesen ist, und liefert mit dem Hinweis auf Dringlichkeit eine adäquate Rechtfertigung für die Handlung der Sprecherin. Der Einschub von halt sorgt für eine stärkere Verknüpfung der beiden Sachverhalte, indem es auf die Vorgängeräußerung rückverweist und somit einen Begründungszusammenhang herstellt: Die unter normalen Umständen abzulehnende Handlung wird als Ausnahme in einem besonderen Fall markiert, wodurch sie begründet akzeptabel wird. Analog zu den Ergebnissen von Blühdorn (2019, S. 301) wird auch hier eine lokale Kohärenzlücke geschlossen, die sich aus der Negativbewertung der eigenen Handlung der Sprecherin aufgetan hat, indem auf eine allgemein zugängliche Wissensbasis zurückgegriffen wird.

Zusätzlich zur inhaltlichen Verknüpfung von Sachverhalten kann halt auch auf (primär) sequenzieller Ebene kohärenzherstellend wirken. Dies zeigt sich am folgenden Beleg, bei dem es sich um einen Ausschnitt aus einer Spielinteraktion handelt. Die Gesprächsteilnehmerinnen sitzen sich gegenüber, können sich aufgrund eines Sichtschutzes aber nicht sehen. Jeder Teilnehmerin liegt ein Blatt Papier vor, auf dem dieselben Gegenstände oder Personen abgebildet sind. Auf dem Blatt von BFD4 ist ein Weg abgebildet, der BFD3 nicht zugänglich ist. Die Aufgabe ist für BFD4, ihrer Gesprächspartnerin den gezeichneten Weg zu erklären, während diese ihn auf ihrem eigenen Papier nachzeichnet. Am Ende werden die Wege verglichen.

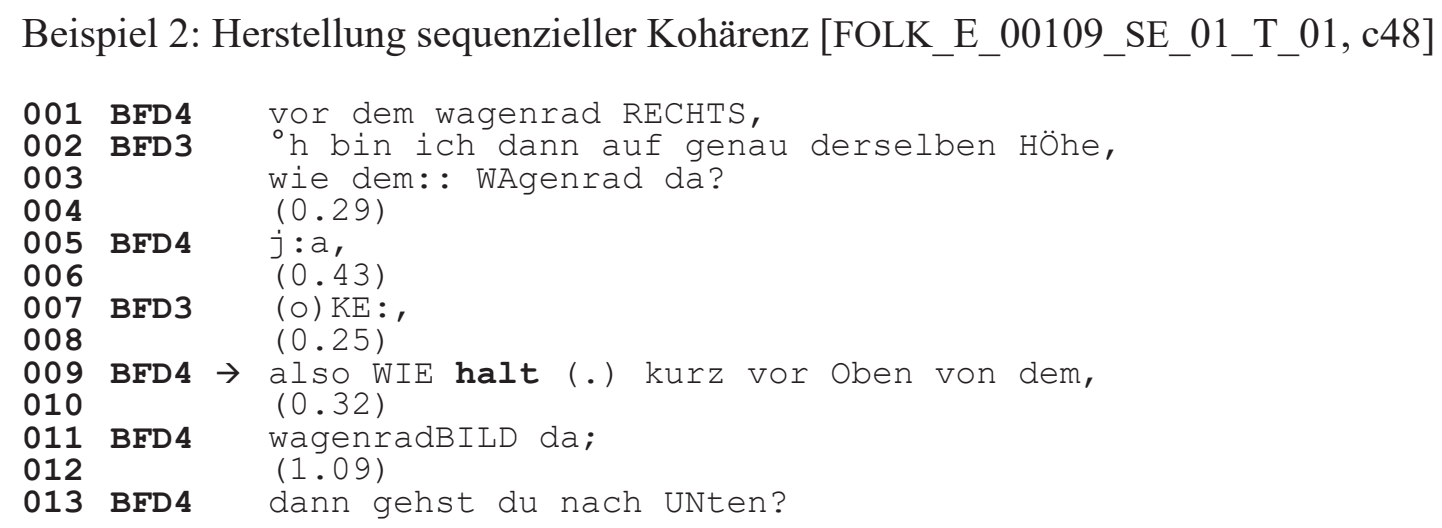

Der gezeigte Interaktionsausschnitt findet zu Beginn des Spiels statt und BFD4 gibt ihrer Gesprächspartnerin die erste Wegbeschreibung. Auf die Instruktion hin, sich vor dem Wagenradbild nach rechts zu wenden, versucht die Rezipientin die Beschreibung zu präzisieren, indem sie die genaue Position erfragt. Dies wird im zweiten Schritt von BFD4 bejaht, woraufhin BFD3 die Sequenz in dritter Position mit einem abschließenden „(o) KE:“ (Z. 007) beendet (vgl. Schegloff 2007, S. 120 ff.). Die formal und sequenziell abgeschlossene Sequenz Frage-Antwort-minimal-post-expansion (Schegloff 2007) wird von BFD4 expandiert, um die Ortsangabe weiter zu präzisieren. Der vorangestellte Diskursmarker also fungiert bereits als Konjunktor, der die Abhängigkeit zum vorhergehenden Bezugsturn markiert (Deppermann/Helmer 2013, S. 13). Die Folgeäußerung stellt eine Spezifikation der Erstinstruktion dar, steht also in Anbindung zum Turn in Zeile 001. Halt markiert dies auf diskursstruktureller Ebene, indem es auf die bereits geäußerte Instruktion zurückverweist und somit beide turns miteinander verknüpft. Durch diese sequenzi- 
elle Bindung markiert die Sprecherin gleichzeitig, dass sie bereits etablierte Informationen wiederholt, wodurch die Instruktion (aufgrund vorheriger Erklärung) als unkompliziert behandelt wird. Anders als im ersten Beispiel geht es hier weniger um die Schließung inhaltlicher Kohärenzlücken zur Verständnissicherung, sondern mehr um die Verbindung der Äußerungen zur Herstellung eines common ground.

Die Abgrenzung inhaltlicher und sequenzieller Kohärenzherstellung ist in den meisten Fällen nur sehr schwer möglich, da beide Aspekte sehr eng aneinander geknüpft sind. Die Verknüpfung einer Äußerung mit einer vorhergehenden Äußerung beinhaltet natürlich auch immer eine gewisse Wiederholung bzw. einen Rückverweis, wodurch automatisch auch Inhalte miteinander verbunden werden. Trotzdem lassen sich Unterschiede in der Verknüpfung festmachen, wie in den vorhergehenden Analysen gezeigt werden konnte. Tendenziell findet sich die (primär) sequenzielle Kohärenzherstellung in den Daten allerdings deutlich seltener als die inhaltliche Verknüpfung von Äußerungen durch halt.

\section{2 halt zum Verweis auf gemeinsames Wissen}

Fischer (2007, S. 63) beschreibt die spezifische Funktion von Modalpartikeln als ,grammaticalised means to refer to the argumentative common ground“. Wie Blühdorn (2019) bereits darlegt, besteht eine zentrale Funktion von halt darin, auf eine gemeinsame Wissensbasis der Interagierenden zu verweisen. Im folgenden Beleg sprechen zwei Lehrer über den vorhergehenden Unterricht von LS, bei dem sein Kollege AN hospitiert hatte und zu dem er ihm nun Rückmeldung gibt. Die Unterrichtsstunde fand in einer Förderklasse statt. Im direkten Präkontext hatte AN Kritik bezüglich der Visualisierungstechniken von LS geäußert, auf die dieser nun eingeht.

Beispiel 3: Verweis auf gemeinsames Wissen [FOLK_E_00144_SE_01_T_01, c169]

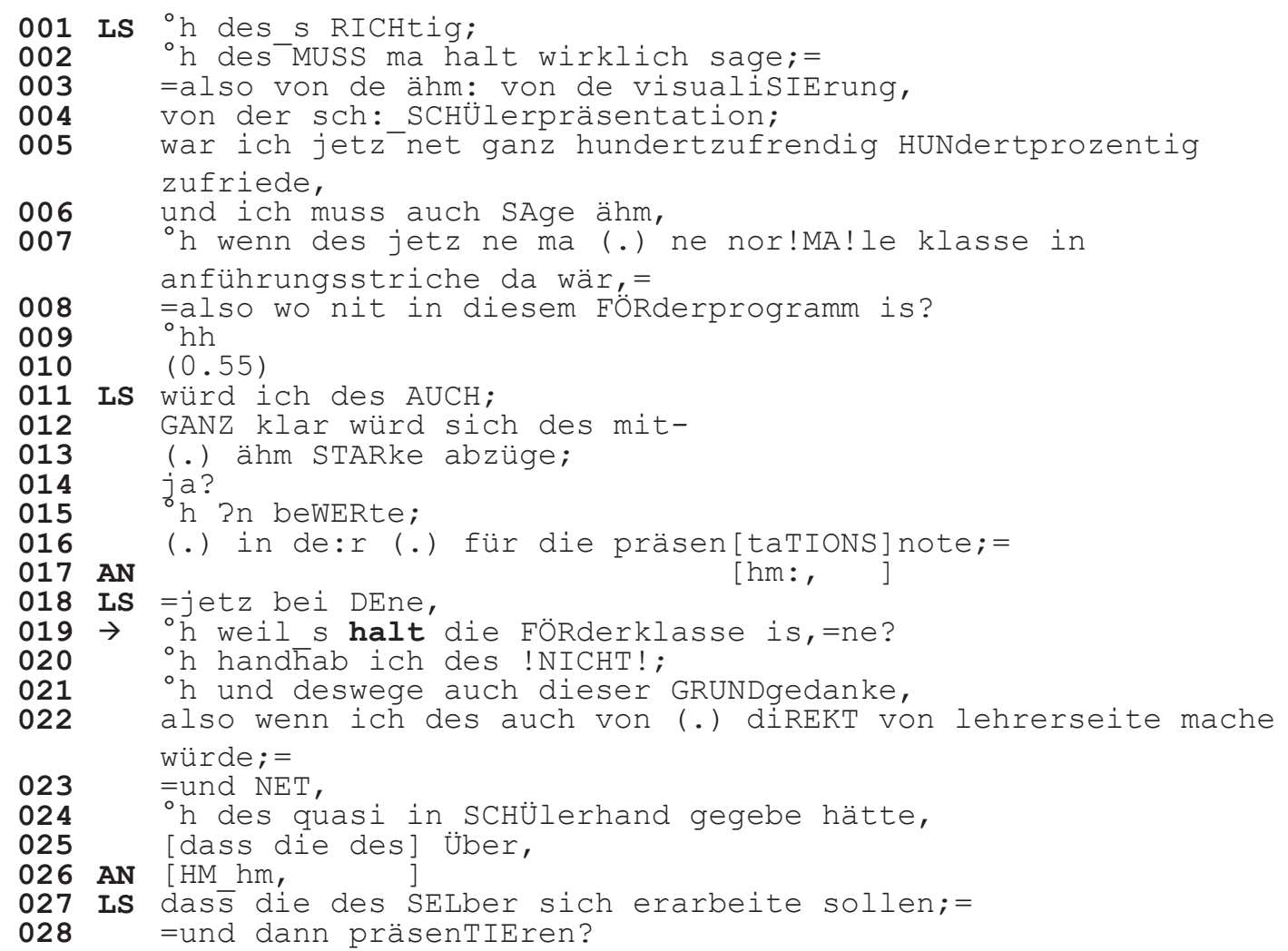


Hier wird halt innerhalb einer Begründungsäußerung realisiert. Der Lehrer LS kontrastiert zunächst die zu berwertende Lerngruppe mit anderen Klassen, indem er die „nor!MA!le[n] klasse[n]“ (Z. 007) von denen ,in diesem FÖRderprogramm“ (Z. 008) abgrenzt. Nachdem er sein hypothetisches Vorgehen bei der Benotung der „normalen Klassen“ darlegt, kommt er auf die tatsächlich zu bewertende Klasse zu sprechen: Die parenthetische Äußerung , ${ }^{\circ} \mathrm{h}$ weil_s halt die FÖRderklasse is,=ne?“ (Z. 019) reaktiviert zunächst die bereits zuvor etablierte Kategorie einer Klasse im Fördeprogramm und setzt sie argumentativ für den Interaktionsverlauf nochmals relevant. Gleichzeitig begründet diese Reaktivierung die Entscheidung, die Schülerpräsentationen nicht in der Bewertung zu berücksichtigen. Diese Begründung wird allein durch die Referenz auf die Kategorie „Förderklasse“ geschaffen, mit der kategoriengebundene Merkmale (im Sinne von category-bound predicates, Watson 1997) ${ }^{14}$ einhergehen, die im Folgenden nicht weiter expliziert werden müssen, weil sie beiden Sprechern gegenwärtig sind. Die Äußerung wird durch den Einschub von halt zu einer plausiblen, konsensfähigen Begründung modalisiert, die keiner weiteren Erläuterung bedarf: Mit halt referiert der Sprecher auf und aktiviert zugleich intersubjektiv vorhandenes (berufsbezogenes) Wissen über das Referenzobjekt (Förderklassen) und seine präsupponierten Merkmale (hier: leistungsschwächer Schüler/innen). Dies wird durch die Kombination mit dem Vergewisserungssignal ne verstärkt, das zusätzlich als Evidenzmarker wirkt und somit den Geltungsanspruch der Bezugsäußerung ebenfalls hervorhebt (vgl. Hagemann 2009). Da der Sprecher bereits zuvor auf den Status der Klasse als Förderklasse verwiesen hat (Z. 008), reaktiviert die halt-Äußerung dieses in der Interaktion verfügbare Wissen und macht es für die Argumentation prospektiv verfügbar und relevant.

Sprecher/innen können einen Sachverhalt durch den Einsatz der Modalpartikel halt als gemeinsame Wissensbasis markieren, wodurch sie ihre Handlungen bzw. Äußerungen für das Gegenüber nachvollziehbar machen können. Die durch die Partikel etablierte Wissensbasis wird als kontextuell ausreichend markiert. Damit operiert die MP auf einer argumentativen Ebene und trägt zur intersubjektiven Ausrichtung der Interagierenden bei. Durch halt wird in Begründungen oder Rechtfertigungen darauf verwiesen, dass der gelieferte Grund für alle Gesprächsteilnehmer/innen evident ist, wodurch die Begründungskraft verstärkt und die Aussage als plausibel markiert wird..$^{15}$ Die Notwendigkeit bzw. die Wahrscheinlichkeit weiterer Ausführungen, Nachfragen oder gar Widerspruch wird herabgesetzt. In den Daten ist auffällig, dass mit halt modalisierte Äußerungen (in dieser Funktion) tendenziell häufiger in längeren Redebeiträgen eines Sprechers/einer Sprecherin auftreten und eher selten in Sequenzen mit vielen Sprecherwechseln. Häufig finden sich (zustimmende) Hörersignale und continuer des Gegenübers (z.B. hm_hm, hm usw.). Dadurch, dass die Partikel halt Äußerungen durch ihre Referenz auf gemeinsames Wissen intersubjektiv als Konsens markiert, wird eine direkte (extensive) Reaktion des Gegenübers oft obsolet.

\section{3 halt zum Informationsmanagement}

Eine wichtige interaktive Aufgabe von Gesprächsteilnehmer/innen ist es, die gegebenen Informationen so zu strukturieren, dass sie für andere nachvollziehbar und verständlich sind. Sprecher/innen verwenden beispielsweise syntaktische Strategien, ,um eine unmar-

\footnotetext{
14 Zur membership categorization analysis allgemein vgl. Sacks (1995).

15 Imo (2008, S. 141) geht davon aus, dass die Plausibilitätsmarkierung eine Grundfunktion von halt darstellt. In meinen Daten zeigt sich ebenfalls, dass Sprecher/innen insbesondere in argumentativen Kontexten durch die Verwendung von halt Argumente oder Sachverhalte als plausibel markieren können.
} 
kierte und damit pragmatisch angemessene Verteilung der Informationen zu erreichen“ (Proske 2013a, S. 207). Die klassischen Untersuchungen zur Informationsstruktur bewegen sich auf einer eher globalen Ebene und fokussieren die Einführung neuer Themen oder Referenten sowie die Orientierung am Informationsstatus der Rezipient/innen (vgl. Lambrecht 1994; Proske 2013a, 2013b). Wenn im Folgenden von informationsstruktureller Funktion gesprochen wird, so ist damit die lokale inhaltliche Strukturierung des Beitrags gemeint (angelehnt an die Arbeiten zur Themenentfaltung und Erzählstruktur, vgl. z.B. Quasthoff 2001).

Der folgende Beleg zeigt einen Ausschnitt aus einer Schichtübergabe in einem Krankenhaus. Eine Mitarbeiterin (ME) berichtet ihrem schichtübernehmenden Kollegen (MJ) von einer Patientin.

Beispiel 4: Markierung von Relevanz [FOLK_E_00114_SE_01_T_01, c512]

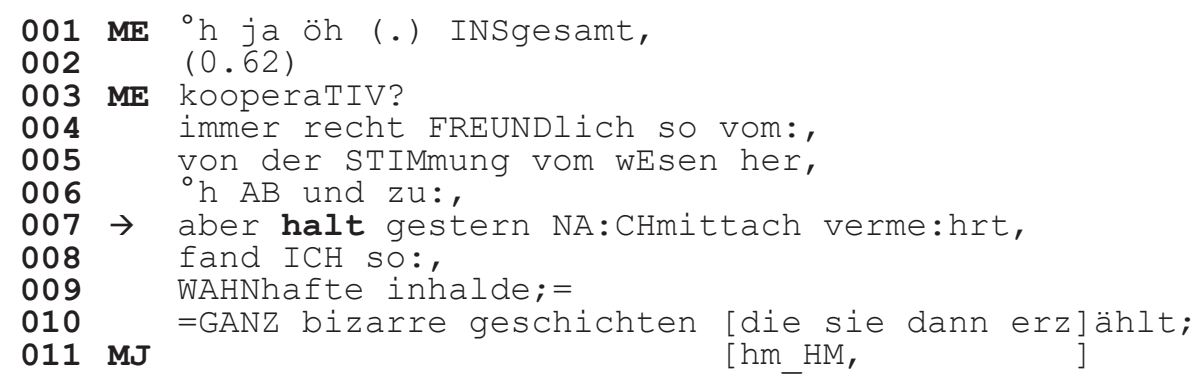

Im Rahmen des Berichts geht die Sprecherin zunächst auf positive Aspekte im Verhalten der Patientin ein, um dann zu den spezifischen Besonderheiten überzuleiten. Für den übernehmenden Kollegen sind vor allem solche Informationen relevant, die ihn auf seine bevorstehende Arbeit vorbereiten. Somit wird durch den kommunikativen Rahmen der Interaktion bereits vorgegeben, dass in erster Linie das Außergewöhnliche erzählenswert (reportable) ist (vgl. Labov 1972). ${ }^{16}$ Der strukturelle und inhaltliche Beginn dieser kontextuell relevanten Informationen wird bereits durch den konzessiven Konjunktor aber projiziert (vgl. Schlobinski 1992, S. 285 ff.): ,,aber halt gestern NA:CHmittach vermeh:rt, fand ICH so:, WAHNhafte inhalde;"“(Z. 007 ff.). Die Platzierung der MP halt in Verbindung mit aber sorgt für eine Relevanzerhöhung des Folgebeitrags, wodurch halt als informationsstruktureller Kontextualisierungshinweis ${ }^{17}$ für den Rezipienten dient. Es zeigt an, dass nun die eigentlich relevante und erzählenswerte Sequenz folgt. Gleichzeitig wird mit halt ein inhaltlicher Bruch markiert, wenn die Mitarbeiterin vom positiven, normalen Verhalten der Patientin zum negativen, ungewöhnlichen Verhalten wechselt, wodurch dieser Aspekt als besonders wichtig hervorgehoben wird. Die Erzählung über die Halluzinationen der Patientin dauert nach diesem Abschnitt noch einige Zeit an, nimmt also viel Gesprächsraum ein, was ebenfalls als Anzeichen für die höhere Inhaltsrelevanz gesehen werden kann.

Interessanterweise kann halt weiterhin als Marker für nicht-zentrale Nebeninformationen dienen. In den meisten Fällen handelt es sich hierbei um Wissenselemente, die für das Verständnis der Erzählung bzw. einzelner Komponenten benötigt werden, für die Gesamterzählung jedoch nebensächlich sind. Anders als im vorherigen Beispiel darge-

\footnotetext{
16 Vgl. auch Ehlich (1983), der das Erzählenswerte im Unerwarteten sieht.

$17 \mathrm{Zu}$ Kontext und Kontextualisierung vgl. Gumperz (1992).
} 
legt, werden die Informationen in dieser Realisierungsvariante also nicht fokussiert, sondern im Gegenteil als sekundär markiert. Im folgenden Beleg erzählt eine Schülerin (NDH4) im Rahmen eines sprachbiografischen Interviews von ihrem Hobby, dem Reiten, und ihrem eigenen Pferd. Auf Nachfrage des Interviewers (NL) berichtet sie vom Kauf des Tieres.

Beispiel 5: Markierung von Nebeninformationen [FOLK_E_00130_SE01_T_02,c315]

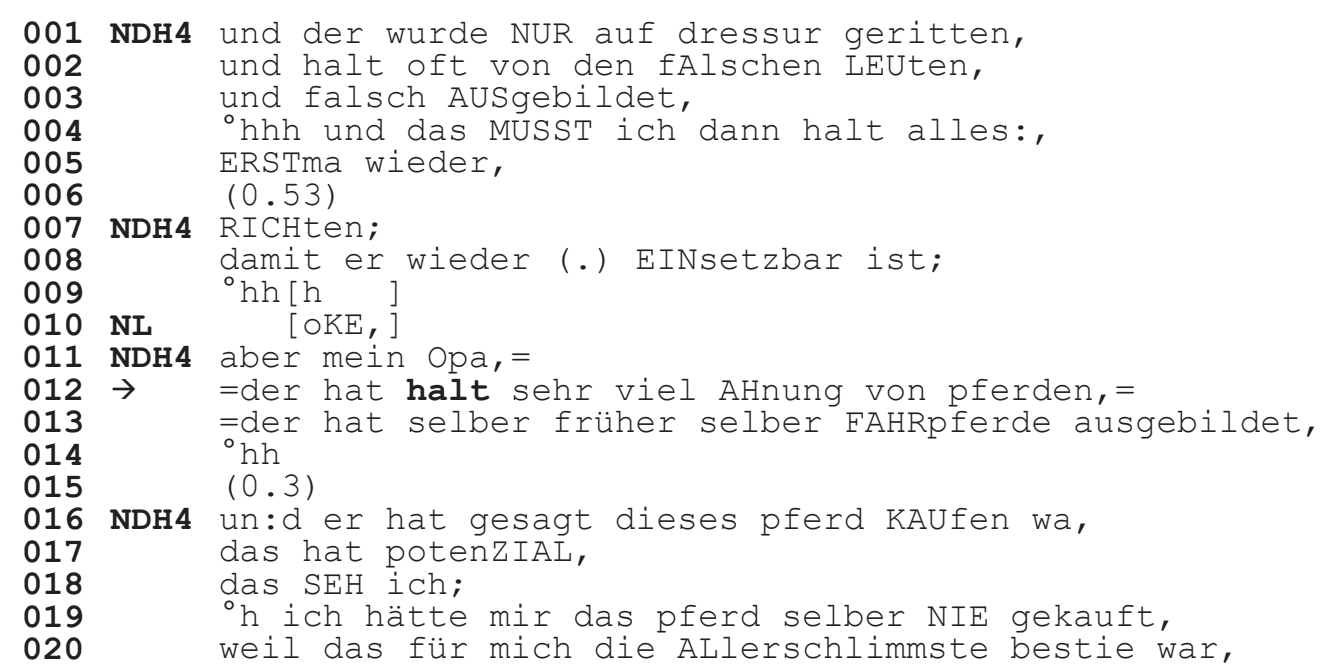

Durch die negative Bewertung des Ausbildungsstands des Pferdes sowie dessen Folgen für die Sprecherin (z.B. hoher Arbeitsaufwand) wird prospektiv eine Erklärung für den Kauf trotz der genannten Gegenargumente relevant gemacht. NL zeigt diese Notwendigkeit durch den continuer „oKE,“ (Z. 010) an, der den vorhergehenden turn durch seine steigende Intonation und sequenzielle Position als unvollständig markiert und somit zur Elaboration auffordert (vgl. Gaines 2011). NDH4 kommt dieser Verpflichtung nach, indem sie ihren Opa als Argument einführt, dessen Einschätzung sie vom Kauf des Pferdes überzeugt hat. Durch die eingeschobene Charakterisierung des Großvaters als jemand, der „halt sehr viel AHnung von pferden“ hat (Z. 012) sowie den direkt mitgelieferten Verweis auf seine Erfahrung als Ausbilder wird er als Experte ausgewiesen. Diese Expertendarstellung ist dem eigentlichen Kaufargument (Empfehlung des Opas) vorgeschaltet und macht die folgende Äußerung projektiv kohärent. Die in der Literatur vordergründig beschriebene Funktion des Verweises auf eine gemeinsame Wissensbasis der Interagierenden durch halt kann an dieser Stelle ausgeschlossen werden, da NDH4 auf Informationen verweist, zu denen sie selbst epistemischen Zugang hat, der Interviewer aber nicht. Vielmehr weist die MP die Sequenz als Einschub sekundär relevanter Informationen aus und wirkt somit als informationsstruktureller Kontextualisierungshinweis. Solche contextualization cues treten selten allein auf, sondern werden in der Regel kombiniert verwendet (Auer 1992, S. 29 f.). So kennzeichnen die schneller werdende Sprechgeschwindigkeit (vgl. das latching) sowie das Einatmen und die kurze Pause im Anschluss (Z. 014, 015) ebenfalls eine Nebensequenz (vgl. Bergmann 2012).

Sprecher/innen nutzen halt rekurrent zum Informationsmanagement, also um längere Gesprächsbeiträge und insbesondere Erzählungen inhaltlich zu strukturieren. Dies ist in vielen Fällen eng verbunden mit der Kohärenzherstellung, die der MP inhärent ist und die Äußerungen so miteinander verknüpft. Der Rückgriff auf eine gemeinsame, allgemein 
zugängliche Wissensbasis kann hierbei größtenteils ausgeschlossen werden, da die erzählten Inhalte im internen Wissensbereich des Sprechers/der Sprecherin liegen und damit für den Rezipienten/die Rezipientin unzugänglich sind; die epistemische Autorität liegt also bei den Sprecher/innen selbst.

\section{Verwendungsweisen der MP eben im gesprochenen Deutsch}

Ebenso wie für halt lassen sich auch für eben drei Funktionsvarianten ausmachen, die ebenfalls nicht als trennscharf anzusehen sind.

\section{1 eben zur Kohärenzherstellung und Gesprächsstrukturierung}

Analog zur funktionalen Gestalt von halt kann auch eben zur Gesprächsstrukturierung qua Kohärenzherstellung eingesetzt werden. Obwohl sich die MPn dabei sehr ähneln, lassen sich doch Unterschiede in der Gebrauchsweise ausmachen: Während halt näher aneinandergrenzende Äußerungen bzw. Sequenzen miteinander verknüpfen kann, wird eben prominent zur Verknüpfung von zeitlich weiter auseinanderliegenden Inhalten eingesetzt, wodurch beispielsweise ein zuvor geäußertes Thema aktualisiert und damit für den folgenden Gesprächsverlauf relevant gesetzt wird.

Das unten stehende Datum entstammt einem Prüfungsgespräch an der Hochschule, wobei $\mathrm{CH}$ als Prüfer und ME als Prüfling agiert. Der erste Transkriptausschnitt zeigt die Frage des Prüfers zum Stichwort „Rezeptions- und Perzeptionsforschung“, die der Prüfling im zweiten Ausschnitt beantwortet. Zwischen den Sequenzen liegen sechs Minuten Gesprächszeit, in der der Prüfling die Antwort vorbereitet und zwischenzeitlich thematisch abschweift, bevor er schließlich zum Stichwort „Perzeptionsforschung“ zurückkommt.

Beispiel 6, Ausschnitt 1: Kohärenzherstellung [FOLK_E_00057_SE_01_T_01, c1021]

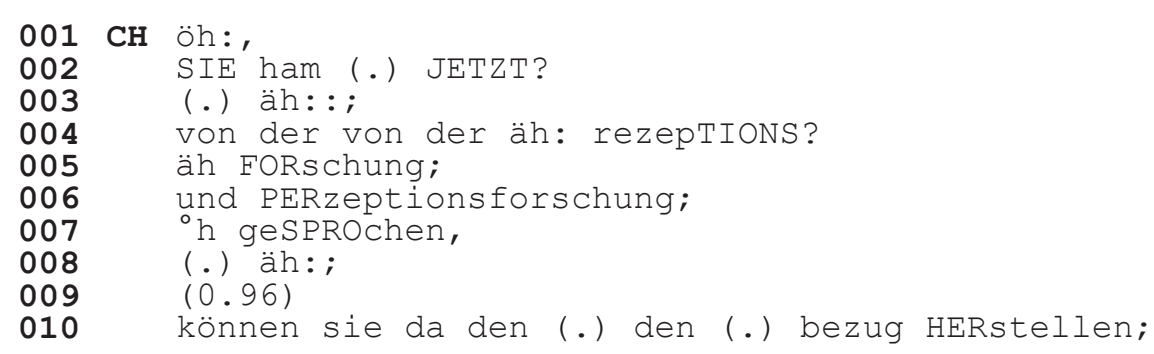

Beispiel 6, Ausschnitt 2: Weiterführung nach sechs Minuten Auslassung

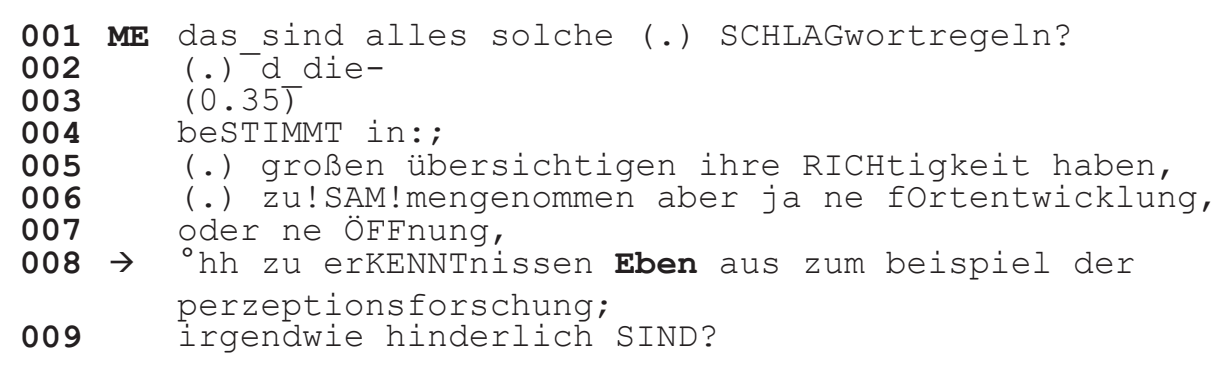

Aufgrund der langen Zwischensequenz, die der Prüfling zur Antwortvorbereitung nutzt, wird es kontextuell relevant, die Rückkehr zur vom Prüfer gestellten Frage zu markieren. Im Rahmen der Prüfungssituation ist es von großer Bedeutung für den Prüfungs- 
kandidaten, auf die Fragen des Prüfers korrekt und wissenschaftlich dezidiert zu reagieren, um Sanktionen zu vermeiden (vgl. Meer 1998). Gemeinsam mit der Wiederholung des relevanten Stichworts ,perzeptionsforschung“ (Z. 008) verweist die MP eben retrospektiv auf das bereits etablierte Thema zurück, wodurch dieses interaktiv aktualisiert wird. Obwohl der Rückverweis auf das vom Prüfer genannte thematische Schlagwort ausreichen würde, ist es im Rahmen der übergeordneten Interaktionsaufgabe notwendig, die prüfungsspezifische Kohärenz (ebd., S. 105) aufrecht zu erhalten, die aufgrund der ausgedehnten Antwortsequenz des Prüflings nicht eindeutig vorhanden ist. In Verbindung mit der MP eben, die zusätzlich zur Wiederholung des Themas einen kontextuellen Rückbezug markiert, wird diese Aufgabe erfolgreich vollzogen. Die MP operiert also auf gesprächsstruktureller Ebene und trägt zur intersubjektiven thematischen Ausrichtung der Interagierenden bei.

Im Vergleich zu halt wird eben häufiger zur Kohärenzherstellung über eine längere Gesprächszeit hinweg eingesetzt, während Sprecher/innen zur kontextnahen Verknüpfung eher auf halt zurückgreifen. Dennoch finden sich in den analysierten Daten ebenfalls solche Verwendungsweisen, in denen eben zur unmittelbaren Strukturierung genutzt wird. Hierbei nutzen Sprecher/innen eben oftmals dann, wenn sie nach einem (thematischen) Einschub in die Hauptsequenz zurückkehren. Im folgenden Beleg beschreibt ein Museumsführer (BB) die Beschaffenheit eines versteinerten Dinosaurierskeletts. Die Gruppe ist direkt vor dem Ausschnitt bei dem neuen Ausstellungsobjekt angekommen.

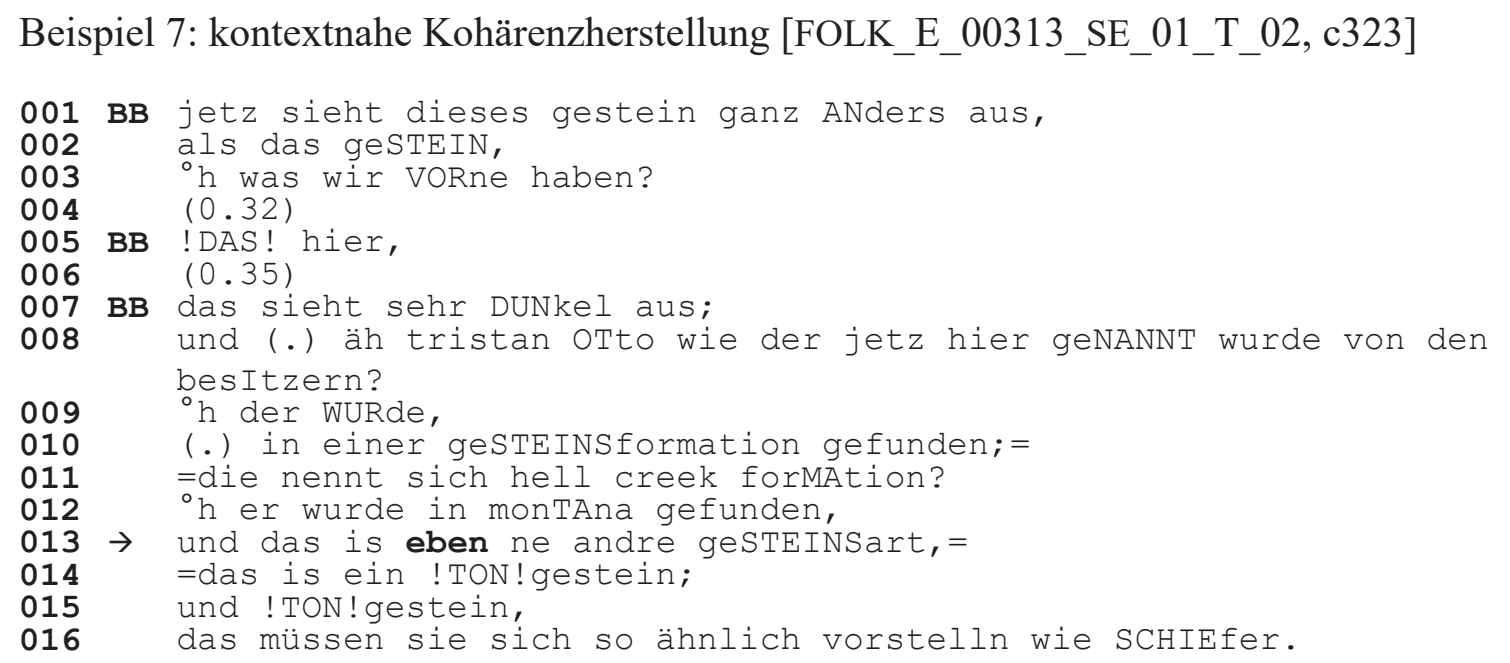

Der Sprecher führt das neue Ausstellungsstück durch eine Kontrastierung zu einem zuvor besprochenen Gesteinstyp ein, indem er beide Skelette miteinander vergleicht. Konkret macht er das Aussehen des Steins zum Thema seiner folgenden Erklärung, indem er auf die Farbe verweist: ,!DAS! hier, (0.35) das sieht sehr DUNkel aus“ (Z. 005-007). Der Konnektor und leitet sodann eine Zwischensequenz ein, in der der Museumsführer den Besucher/ innen Hintergrundinformationen zum Skelett, dessen Namen und Fundort gibt (Z. 008012). Diese Digression vom übergeordneten Thema des Gesteins an sich gibt BB die Möglichkeit, spezifisch auf das Dinosaurierskelett einzugehen, indem er es konkret benennt (,tristan OTto“, Z. 008) und den genauen Fundort darlegt. Dieser kurzzeitige Fokuswechsel (Kallmeyer 1987) wird durch den Pronomenwechsel (das zu der/er) markiert, resultierend aus dem Wechsel des Subjekts. Der Fokuswechsel hat in diesem Fall die Funktion, Zusatzinformationen zum betrachteten Objekt zu liefern, ohne aus der wissensvermittelnden 
Sequenz auszusteigen. Die Rückkehr in die Erklärungssequenz zum Gestein an sich erfolgt wiederum durch die Wiederaufnahme des Pronomens das sowie durch die MP eben: „und das is eben ne andere geSTEINSart,= =das is ein !TON!gestein" (Z. $013 \mathrm{f}$.). Eben markiert hier den Rückbezug zur begonnenen Beschreibung des Gesteins als „DUNkel“ (Z. 007), stellt also retrospektiv einen kohärenten Zusammenhang zwischen den Turnkonstruktionseinheiten her. Der Sprecher verknüpft die beiden Teile der Hauptsequenz miteinander und beendet somit seine begonnenen Erläuterungen zur Spezifikation des Gesteins: Der Stein sieht anders aus, als die vorher gesehenen Steine, was daran liegt, dass es sich um eine andere Gesteinsart handelt. Der abrupte Pronomenwechsel sowie der Fokussprung zurück in die Hauptsequenz sind für die Zuhörer/innen nachvollziehbar und unmarkiert. ${ }^{18}$ Damit trägt die MP dazu bei, den längeren Gesprächsbeitrag des Museumsführers so zu strukturieren und zu organisieren, dass er reibungslos Erklärungen auf verschiedenen Ebenen liefern kann, ohne dabei inkohärent zu werden und Unverständnis seitens der Zuhörer/ innen zu riskieren. Gleichzeitig wird durch den Rückverweis auf die Zeilen 001-007 der Argumentationszusammenhang zusammengehalten, sodass die zu Beginn implizit vorhandene Frage, wieso der Stein denn anders aussieht, kohärent vom Sprecher aufgelöst werden kann.

Diese Funktion kann in den Daten überwiegend in längeren monologischen Sequenzen nachgewiesen werden, beispielsweise in Antwortsequenzen innerhalb universitärer Prüfungsgespräche oder längeren Argumentationssequenzen in Diskussionen. Die MP eben stellt somit eine Ressource für die Sicherung einer intersubjektiven Verstehensbasis im Rahmen längerer monologischer Beiträge dar. Blühdorn (2019, S. 301) beschreibt solche Verwendungsweisen als „Schließung einer Kohärenzlücke [...], die sich in der aktuellen (Inter-)Aktion aufgetan hat". Ich würde aufbauend auf den vorangegangenen Analysen dafür argumentieren, dass eben außerdem dafür eingesetzt werden kann, solche Kohärenzlücken in der Interaktion gar nicht erst aufkommen zu lassen, indem von vornherein Zusammenhänge als solche markiert werden.

\subsection{Argumentative Funktionen von eben}

Ähnlich wie die MP $j a$, die oftmals die Aufgabe einnehmen kann, ,,in Argumentationen kollektiv Strittiges über etwas kollektiv Geltendes unstrittig zu machen“ (Reineke 2016, S. 120), wird auch eben zur Modalisierung geltender Argumente bzw. Schlussfolgerungen genutzt. Die Daten zeigen hierbei zwei zentrale Verwendungsweisen der MP, i) die Herstellung von Evidenz insbesondere in schlussfolgernden Aussagen und ii) eine verstärkende Funktion durch die Partikelkombination eben nicht.

\subsubsection{Zur Markierung von Evidenz}

Thurmair (1989, S. 119) stellt die Markierung von Evidenz als eine zentrale Funktion von eben heraus und argumentiert, dass Sprecher/innen Sachverhalte so als gegeben markieren können. Dies findet sich auch in den vorliegenden Daten als rekurrente Verwendungsweise der MP.

Der folgende Gesprächsausschnitt aus einer öffentlichen Podiumsdiskussion im Rahmen der Stuttgart-21-Schlichtungsgespräche illustriert dies exemplarisch.

18 Die Teilnehmergruppe zeigt keinerlei Anzeichen für Unverständnis oder Passungsprobleme an - weder auf verbaler Ebene noch auf gestisch-mimische Art und Weise, wie im Video zu sehen ist. 
Beispiel 8: Markierung von Evidenz [FOLK_E_00064_SE_01_T_09, c389]

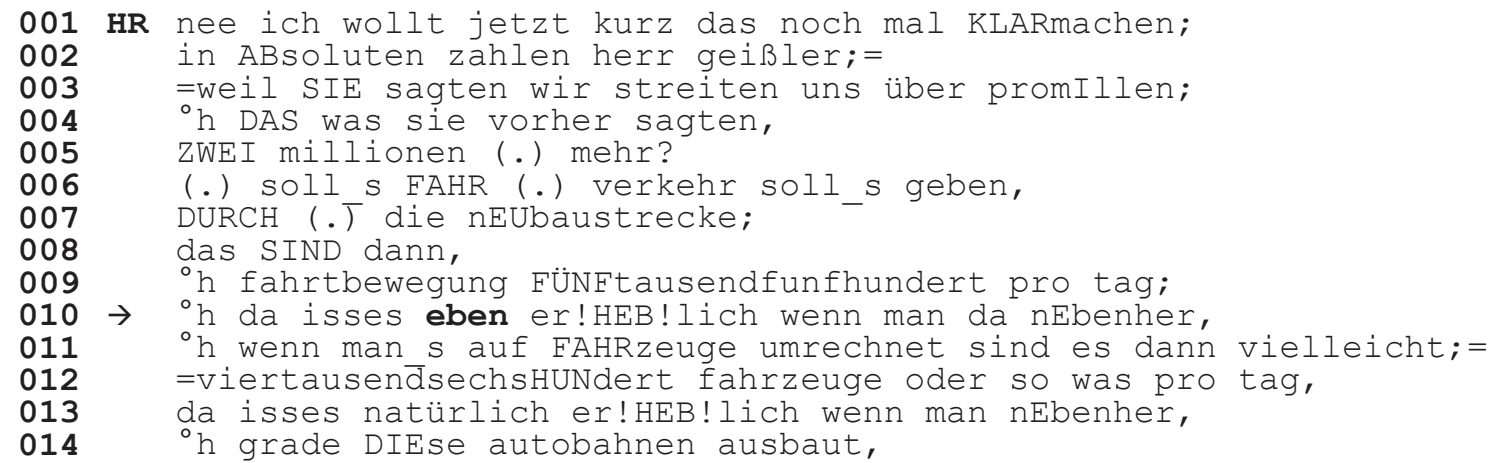

Innerhalb seiner Argumentation rechtfertigt sich HR gegenüber eines vorherigen Vorwurfs des Moderators HG, man würde sich aktuell ,über Promillen“ streiten. Er erörtert zunächst absolute Zahlen, bevor er schließlich zur schlussfolgernden Widerlegung ansetzt, dass das besprochene Thema gerade nicht unerheblich ist („da isses eben er!HEB!lich wenn man da nEbenher,“, Z. 010). HR unterbricht seine Aussage, um weitere Zahlen zu nennen und führt seine Konklusion schließlich mit einer Reformulierung zu Ende: „da isses natürlich er!HEB!lich wenn man nEbenher, 'h grade DIEse autobahnen ausbaut,“(Z. 013 f.). Eben markiert die zentrale Schlussfolgerung des Sprechers, die den vorhergehenden Vorwurf der Irrelevanz widerlegen soll, und modalisiert sie als für alle Beteiligten offensichtliches Wissen. Dies wird besonders durch die Reformulierung in Zeile 014 unterstützt, wenn der Sprecher eben durch natürlich ersetzt und somit auf deutlich erkennbare Art und Weise auf die Evidenz seiner Widerlegung hinweist.

Durch eben werden häufig solche Aussagen markiert, die als zentral gesehen werden können, also beispielsweise Argumente widerlegen oder Schlussfolgerungen darstellen (vgl. hierzu auch Rost-Roth 1998). Durch die evidenzmarkierende Funktion der MP wird der Geltungsanspruch der Äußerungen gehoben, wodurch sie im Gesprächszusammenhang eine höhere Relevanz erhalten. Somit wird eben als argumentativ-strategisches Element zur Stärkung der eigenen Argumentation genutzt. ${ }^{19}$

\subsubsection{Zur Verstärkung}

In den vorliegenden Daten zeigt sich die Kombination eben nicht als häufige Realisierungsmöglichkeit zur argumentativen Verstärkung. Der folgende Beleg ist erneut aus einem der Stuttgart-21-Schlichtungsgespräche entnommen.

19 Thurmair (1989, S. 123) weist darauf hin, dass eben aufgrund seiner evidenzmarkierenden Funktion ein „Mittel des Argumentierens [ist], das über problematische oder noch zu problematisierende Sachverhalte hinwegtäuscht bzw. hinwegtäuschen kann“. Sie argumentiert, dass eben-Äußerungen rechthaberisch wirken können, da der Sprecher/die Sprecherin den betreffenden Sachverhalt mit der Herstellung von Evidenz als nicht erklärungsbedürftig einstuft. Diese zugeschriebene Konnotation konnte in den vorliegenden Daten nicht nachgewiesen werden, insbesondere da die Wirkung einer Äußerung auf das Gegenüber nur schwer nachzuvollziehen ist. Es konnten keine Aufzeigeleistungen der Rezipient/innen gefunden werden, die auf eine rechthaberische Interpretation der eben-Äußerung hindeuten würden. Stattdessen zeigt sich, dass durch die Realisierung von eben Schlussfolgerungen von meist ausführlichen Argumentationsverläufen gekennzeichnet werden, deren Gültigkeitsstatus zum Zweck der Verstärkung hervorgehoben wird. Zudem zeigt Beispiel 8 vor der eben-Äußerung eine längere, explizite Begründung bzw. Erklärung des Sachverhalts (ein Muster, das sich häufiger zeigt), wodurch die von Thurmair beschriebene Funktion der Markierung als nicht erklärungsbedürftig widerlegt werden konnte. 
Beispiel 9: Verstärkung [FOLK_E_00064_SE01_T_06, c345]

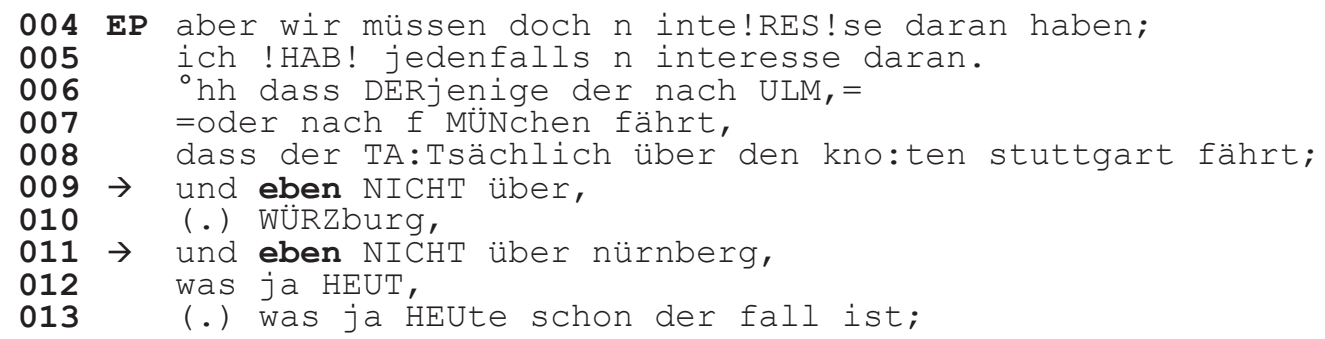

Es geht hier um die Neubaustrecke Wendlingen-Ulm, für deren Ausbau der Sprecher Ernst Pfister (EP) argumentiert. Dabei kontrastiert er die Möglichkeiten für Reisende, statt über Würzburg oder Nürnberg über Stuttgart nach München zu fahren, wobei zweimal die Partikelkombination eben nicht vorkommt (Z. 009, 011). Durch die kohärenzherstellende Funktion von eben wird ein Rückbezug zur vorhergehenden Interaktionsgeschichte markiert, während die prosodische Hervorhebung der Negationspartikel nicht verstärkend wirkt. Der Sprecher markiert hiermit, dass ebendiese Fahrtalternativen für die Zukunft unattraktiver werden müssen. Rhetorisch bewirkt dies eine Präzisierung der Argumentation, wodurch die Position des Sprechers untermauert wird.

In Gesprächen, in denen Argumentieren als zentrale Handlung im Vordergrund steht, stellt die Herstellung der Glaubwürdigkeit der eigenen Argumente und Schlussfolgerungen eine zentrale Interaktionsaufgabe dar (vgl. Deppermann 1997; Spranz-Fogasy 2005). Interagierende müssen stets dafür sorgen, dass ihre Argumentation nachvollziehbar und unstrittig formuliert und vor allem vom Gegenüber aufgenommen wird. „Argumentation beruht darauf, daß unterstellt wird, daß gewisse Überzeugungen und Kriterien unstrittig sind“" (Deppermann 1997, S. 39). Die Markierung von Offensichtlichkeit sowie die Verstärkung bestimmter Aspekte sind hierbei wichtige Formate, die den Gesprächsteilnehmer/innen zur Verfügung stehen. Die Analysen zeigen, dass die Modalpartikel eben eine rekurrent genutzte lexikalische Ressource darstellt. Daher ist es nicht verwunderlich, dass ein Großteil der Vorkommen von eben (87\%) in institutioneller bzw. öffentlicher sowie in handlungsorientierter Alltagsinteraktion nachgewiesen wurde (Prüfungs- und Schlichtungsgespräche, Interviews, Lehr-Lern-Kontexte und Spieleinteraktionen) - also überall dort, wo Sprecher/innen ein (kommunikatives) Ziel verfolgen, das durch verbale Aushandlungen erreicht werden kann.

\section{5. $\quad$ Es ist halt eben nicht dasselbe - zur Frage der Ersetzbarkeit und Synonymie von halt und eben}

Wie eingangs bereits kurz erwähnt, wird in der Literatur oftmals ein synonymes Verhältnis der MPn eben und halt angenommen (Weydt 1969; Hentschel 1986; Thurmair 1989; Dittmar 2000; Kwon 2005). Kwon (2005, S. 66) geht beispielsweise davon aus, dass

eben fast immer durch halt ohne den Verlust oder die Hinzufügung einer Bedeutung ersetzbar [ist]. Ob halt umgekehrt durch eben ersetzbar ist, scheint aber von dem Kontext abhängig zu sein, weil sich die Partikel eben im Gegensatz zu halt in verschiedenen syntaktischen Funktionen realisieren lässt. ${ }^{20}$

20 Kontextabhängigkeit meint hier die syntaktische Umgebung und die entsprechenden Funktionstypen. 
Thurmair (1989) spricht nicht von Synonymie, sondern von einer grundlegenden Bedeutungsähnlichkeit beider Partikeln. Als Argument gegen eine Synonymie nennt sie ,ihr unterschiedliches Verhalten in den Kombinationen [...] und die Tatsache, daß sie miteinander kombiniert werden können (echt synonyme Partikeln wie z. B. eh und sowieso werden [...] nie kombiniert)“" (Thurmair 1989, S. 124, Hervorhebung i. O.; vgl. auch Thurmair 1991).

Durch die Analyse authentischer Alltagsinteraktionen unter interaktionslinguistischen Aspekten konnte in diesem Beitrag herausgearbeitet werden, dass beide Partikeln durchaus funktionale Gemeinsamkeiten aufweisen. Beide Partikeln verfügen sowohl über epistemische als auch strukturierende Elemente; sie können beide zum Verweis auf einen kontextuellen Prätext eingesetzt werden und somit Kohärenz innerhalb eines Beitrags bzw. turnübergreifend herstellen. Sie bewegen sich also auf einem Spektrum zwischen intersubjektivem Wissensmanagement und Beitrags- bzw. Gesprächsstrukturierung.

Dennoch zeigen sich bei detaillierter Betrachtung distinktive Verwendungsweisen der jeweiligen Partikeln, die gegen eine grundsätzliche Austauschbarkeit oder sogar Synonymie sprechen. Zunächst werden eben und halt zwar beide zur Kohärenzherstellung eingesetzt, jedoch zeigen sich sequenziell und kontextuell unterschiedliche Verwendungsweisen: Halt wird tendenziell eher für eine kontextnahe Kohärenzherstellung genutzt, während mit eben Verknüpfungen über längere Gesprächszeiten und zum Teil sogar über mehrere Einzelgespräche hinweg markiert werden können. Mit halt greifen Sprecher/innen oft auf eine allgemein verfügbare Wissensbasis zurück, wodurch sie Äußerungen, Handlungen, Intentionen usw. miteinander in Verbindung setzen und so für das Gegenüber nachvollziehbar machen. Im Gegensatz dazu greift eben auf eine gesprächsinterne, also nicht allgemein verfügbare Wissensbasis zurück, wenn es auf einen bereits etablierten Prätext verweist. Zudem kann eben als mehr oder weniger unmittelbar verknüpfendes Element innerhalb eines längeren Turns dienen, um beispielsweise nach einer Nebensequenz wieder zurück zur Hauptsequenz zu finden. Im Gegensatz zu halt wirkt eben nicht prospektiv, sondern nur retrospektiv kohärenzherstellend.

Wenn Thurmair von einer grundsätzlichen Ersetzbarkeit von eben und halt spricht, so argumentiert sie mit den partikelinhärenten unterschiedlichen Graden des Geltungsanspruchs:

Überspitzt könnte man vielleicht sagen: halt kann immer dann nicht durch eben ersetzt werden, wenn das Plausible nicht evident ist; da aber umgekehrt das Evidente (in der Regel) auch plausibel ist, ist erklärt, warum eben immer durch halt zu ersetzen ist. (Thurmair 1989, S. 128, Hervorhebung i. O.)

Meines Erachtens ist nicht der Grad des Geltungsanspruchs entscheidend für die jeweilige Partikelfunktion (und damit ihrer möglichen Ersetzbarkeit), sondern der Gesprächskontext, insbesondere der Präkontext. Aufgrund der Konnexfunktion beider Partikeln, die sich jedoch leicht voneinander unterscheiden, sind die vorhergehenden Gesprächsschritte für die Bedeutungs- bzw. Funktionsanalyse hochgradig relevant. So sind die von Thurmair und anderen Autor/innen (oftmals ausgedachten) Beispiele für die Unersetzbarkeit von halt durch eben in den meisten Fällen durch die Vorstellung eines entsprechenden Präkontextes widerlegbar. Nach Thurmair (1989, S. 124) sind die MPn folgend nicht austauschbar:

(1) „Du kannst deine Freunde schon mitbringen.

Wir haben halt kein Bier mehr.

*Wir haben eben kein Bier mehr.“ 
In einem Gesprächsszenario, in dem die Interagierenden vorher darüber gesprochen haben, dass der Biervorrat langsam knapp wird, ist der Gebrauch von eben als kohärenzherstellendes Element durchaus vorstellbar. Umgekehrt spricht Thurmair vom inadäquaten, aber nicht unmöglichen Gebrauch von halt in folgenden Beispielen:

(2) „Der Wal ist eben ein Säugetier. vs. ?Der Wal ist halt ein Säugetier.

Der Krieg ist eben unmoralisch. vs. ?Der Krieg ist halt unmoralisch.“

Der Unterschied liegt hier nicht im Geltungsanspruch der jeweiligen Aussage, sondern in der Konnexfunktion: Die eben-Äußerungen wirken argumentativer, während die haltÄußerungen auf eine gemeinsame Wissensbasis zu referieren scheinen, also auf epistemischer Ebene operieren. Je nach Gesprächskontext sind beide Varianten akzeptabel, unterscheiden sich allerdings in ihrer Funktion. ${ }^{21}$

Über die Ersetzbarkeit beider Partikeln hinaus wird in der Literatur häufig von ihrer Kombinierfreudigkeit gesprochen (z. B. Thurmair 1989, 1991; Dittmar 2000; Müller 2016, 2018). Während Thurmair (1989) die Kombination eben halt als ungrammatisch einstuft, wird sie von Dittmar (2000) in seiner Untersuchung von Berliner Sprachdaten um die Wende auffällig häufig nachgewiesen. Er konstatiert, dass die Kombinationsreihenfolge davon abhängig ist, welche MP in der jeweiligen Region Deutschlands überwiegt, die sodann von der jeweils anderen ergänzt werden kann. Für die Kombination eben halt nimmt er eine Abtönung des stärkeren eben durch das „weichere“ halt an (vgl. ebd., S. 224). Demgegenüber geht Müller (2016) unabhängig von der Realisierungsreihenfolge von einer Addition beider Partikelbedeutungen aus.

In den vorliegenden Stichproben finden sich in den insgesamt 167 untersuchten Partikelvorkommen lediglich drei Kombinationen beider Partikeln (1x eben halt, $2 \mathrm{x}$ halt eben) ${ }^{22}$

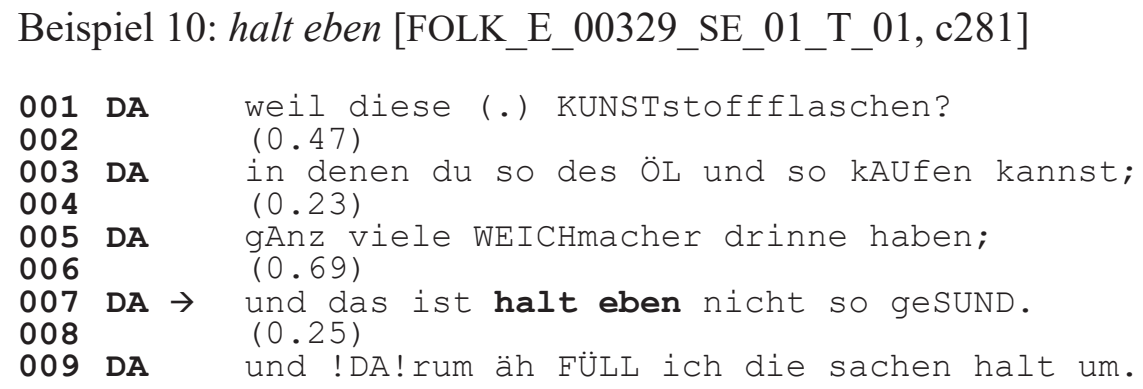

Der Beleg stammt aus einer Tupperparty, bei der eine Beraterin (DA) in einem mehr oder weniger privaten Kontext Tupperware-Produkte vorstellt, um diese an die Teilnehmer/ innen zu verkaufen. Die Verkäuferin erwähnt, dass sie Produkte wie Ö1 und Essig in Glasflaschen umfüllt, woraufhin sie nach ihren Gründen gefragt wird, die sie nun erläutert. Sie

21 Selbstverständlich sind diese Interpretationen spekulativ und anhand einzelner, kontextloser Äußerungen nicht nachweisbar. Dennoch lässt sich auf der Grundlage der in diesem Beitrag durchgeführten Analysen zeigen, dass die bisher postulierte Ersetzbarkeit (bzw. Nicht-Ersetzbarkeit) von halt und eben nicht so einfach zu begründen ist, wie bisher dargestellt. Beide MPn werden von Interagierenden systematisch in spezifischen Kontexten genutzt, um spezifische Funktionen zu erfüllen, die nicht lediglich den Grad der Verbindlichkeit betreffen.

22 Eine DGD-Recherche über alle Vorkommen hinweg zeigt jedoch 75 Treffer für halt eben und neun Treffer für eben halt. Dies deckt sich mit den bisherigen Annahmen, dass halt eben die häufigere Kombinationsmöglichkeit darstellt. Müller (2018, S. 479, Hervorhebung i. O.) spricht von Markiertheitsunterschieden und stuft „die Abfolge halt eben als unmarkiert und eben halt als markiert ein“. 
argumentiert, dass die handelsüblichen Verpackungen Weichmacher enthalten ,und das ist halt eben nicht so geSUND“ (Z. 007). Die Funktion von halt ist in diesem Fall der Verweis auf eine allgemein zugängliche Wissensbasis, wodurch die Proposition als allgemein unstrittig markiert wird. Das folgende eben wirkt auf argumentativer Ebene zusätzlich evidenzmarkierend und hebt dadurch die Geltung des Arguments hervor. Beide Partikeln wirken sich auf den Geltungsanspruch des Arguments aus, welcher somit stark zunimmt.

Darauf aufbauend schließe ich mich Thurmair $(1989,1991)$ sowie Müller (2018, S. 272) an, die von einer additiven Verknüpfung der Modalpartikelfunktionen ausgehen. Grundsätzlich setzt die Partikelkombination den Geltungsanspruch herauf, womit eine argumentative Verstärkung einhergeht. Trotzdem können einzelne Funktionskomponenten der MPn identifiziert werden, was ein weiteres Argument gegen die pauschal mögliche Ersetzbarkeit bzw. Synonymie von halt und eben ist.

\section{6. $\quad$ Fazit}

Die in der Literatur häufig im Zusammenhang mit eben und halt postulierten Konnotationen von Unabänderlichkeit oder Resignation (vgl. z. B. Helbig 1988) konnten in den vorliegenden Daten interessanterweise nicht nachgewiesen werden. Bei den Vorkommen im Korpus, bei denen eine leichte Tendenz in Richtung des Ausdrucks von Unabänderlichkeit festgestellt werden kann, handelt es sich um generische Aussagen (z. B. is halt so, das war halt eben früher so). In solchen Fällen scheint weniger die lexikalische Bedeutung der Partikeln zur Konnotation unabänderlich zu führen, sondern der jeweilige (Äußerungs-)Kontext, in den die Partikel eingebettet ist. In Anlehnung an Deppermann/Elstermann (2008) sowie Deppermann (2009) kann also von verschiedenen Quellen für die Bedeutungskonstitution gesprochen werden: Die häufig aufgeführte Funktion von eben und halt Unabänderlichkeit und Resignation auszudrücken ist nicht der partikelinhärenten Bedeutung zuzuschreiben, sondern sie entsteht durch spezifische Verwendungskontexte; es handelt sich also um eine äußerungs- oder interaktionskontextspezifische Bedeutung. Werden generische oder gar tautologische Aussagen mit eben oder halt markiert, werden die lexemspezifischen Merkmale aktiviert, die zu einer Heraufsetzung der Gültigkeit der Aussagen führen. Äußerungen wie is halt so beinhalten bereits ganz ohne die MP einen propositionalen Unveränderlichkeitsaspekt. ${ }^{23}$ Ein Ausdruck von Sprecherresignation konnte im zugrundeliegenden Korpus unter Berücksichtigung kontextueller Faktoren nicht nachgewiesen werden. Diese Interpretation ist meines Erachtens ein Resultat aus der äußerungsspezifischen Bedeutung von Unabänderlichkeit, mit der ein gewisser Fatalismus einhergeht.

Die in diesem Beitrag vorgelegten Analysen bestätigen die anfängliche These, dass es sich bei den MPn halt und eben um jeweils selbstständige Partikeln mit eigenem Funktionsspektrum handelt, die individuell, statt als austauschbare Quasisynonyme betrachtet werden müssen. Trotz ihrer funktionalen Parallelen zeigen sie distinktive Verwendungsweisen, auf die von Interagierenden im Rahmen von (Gesprächs-)Strukturierung, Wissensmanagement und Argumentationen systematisch zurückgegriffen wird. Während halt Beiträge eher auf informationsstruktureller Ebene gliedern kann, wird eben strategisch-argumentativ innerhalb von konfliktären oder begründungsrelevanten Interaktionen genutzt, um Widersprüche zu erschweren und so einen kommunikativen Vorteil

23 Eventuell könnte man im Fall von das ist halt/eben/halt eben so sogar von einer konstruktionsspezifischen Bedeutung sprechen. Da in diesem Beitrag jedoch nicht von einem konstruktionsgrammatischen Ausgangspunkt argumentiert wurde, sei dies nur am Rande erwähnt. 
zu schaffen. Beides sind flexible kommunikative Ressourcen, die in verschiedenen Verwendungskontexten systematisch zur Lösung spezifischer Interaktionsaufgaben wie Beitragsstrukturierung, Wissensmanagement und Überzeugungsarbeit genutzt werden. Zudem konnte herausgearbeitet werden, dass die vielfach postulierte Substituierbarkeit oder sogar Synonymie beider Partikeln nicht haltbar ist. Detaillierte turn-by-turn-Analysen zeigen jeweils spezifische usuelle Verwendungen, die nicht von der jeweils anderen Partikel übernommen werden. Interagierende nutzen halt und eben rekurrent in unterschiedlichen Gesprächs-, Sequenz- und Äußerungskontexten zur Bearbeitung ähnlicher und unterschiedlicher kommunikativer Aufgaben; eine pauschale Zusammenfassung beider Partikeln würde die Interaktionsrealität daher nicht adäquat widerspiegeln.

Aus dieser Untersuchung lassen sich abschließend einige grundlegende Punkte bezüglich der Analyse von Modalpartikeln herleiten. Zunächst ist grundsätzlich die Gültigkeit von kontextlosen Ersetzungsproben sowie Bedeutungs- und Funktionszuschreibungen unzulänglich, u. a. aufgrund denkbarer Präkontexte. Folglich ist die Analyse im authentischen Sequenzkontext unumgänglich, um adäquate, die Verwendungsrealität abbildende Aussagen über Partikelbedeutung und -funktion treffen zu können. Daher ist eine dezidierte Betrachtung der MPn nötig, d.h. i) in ihrer natürlichen Umgebung (authentische (Alltags-) Interaktionen), ii) in Verbindung mit dem sequenziellen Kontext, in den sie eingebettet sind, und iii) in ihrem spezifischen Äußerungskontext. Weiterhin scheint es besonders bei multifunktionalen Partikeln wichtig zu sein, das Verhältnis von Partikelbedeutung, Sequenzkontext und Äußerung zu beachten, die in der Funktionskonstitution oftmals eng ineinander greifen.

In diesem Sinne könnte in weiteren Forschungsarbeiten überprüft werden, inwieweit die hier eruierten Funktionen der Mordalpartikeln mit interaktions- bzw. gattungsspezifischen constraints wie etwa Dialogizität und Monologizität, wissensvermittelnden oder argumentativen Kontexten oder Erzählsequenzen verbunden sind. Hierbei wäre es lohnenswert, die Stichprobengröße der Studie zu erhöhen, um eine quantitative Überprüfung der dargestellten Ergebnisse zu erzielen.

\section{Literatur}

Auer, Peter (1992): Introduction: John Gumperz' approach to contextualization. In: Di Luzio, Aldo/Auer, Peter (Hg.): The contextualization of language. Amsterdam u. a.: Benjamins. S. 1-37. (= Pragmatics \& Beyond New Series 22).

Bergmann, Pia (2012): The prosodic design of parentheses in spontaneous speech. In: Bergmann, Pia/Brenning Jana/Pfeiffer, Martin/Reber, Elisabeth (Hg.): Prosody and embodiment in interactional grammar. Berlin: De Gruyter. S. 103-141. (= linguae \& litterae 18).

Betz, Emma/Deppermann, Arnulf (2018): Indexing Priority of Position: Eben as Response Particle in German. In: Research on Language and Social Interaction 51. S. 171-193.

Blühdorn, Hardarik (2019): Modalpartikeln und Akzent im Deutschen. In: Linguistische Berichte 259. S. 276-317.

Brauße, Ursula (1986): Zum Problem der sogenannten Polyfunktionalität von Modalpartikeln. Ja und eben als Argumentationssignale. In: Zeitschrift für Phonetik, Sprachwissenschaft und Kommunikationsforschung 39, 2. S. 206-223.

Brown, Penelope/Levinson, Steven (1987): Politeness. Some universals in language usage. Cambridge: Cambridge University Press. (= Studies in Interactional Sociolinguistics 4).

Brünjes, Lena (2014): Das Paradigma deutscher Modalpartikeln. Dialoggrammatische Funktion und paradigmeninterne Oppositionen. Berlin u. a.: De Gruyter. (= Reihe Germanistische Linguistik 301). 
Deppermann, Arnulf(1997): Glaubwürdigkeit im Konflikt. Rhetorische Techniken in Streitgesprächen; Prozeßanalysen von Schlichtungsgesprächen. Frankfurt a. M.: Lang. (= Europäische Hochschulschriften: Reihe 21, Linguistik 184).

Deppermann, Arnulf (2009): Verstehensdefizit als Antwortverpflichtung: Interaktionale Eigenschaften der Modalpartikel ,denn` in Fragen. In: Günthner, Susanne/Bücker, Jörg (Hg.): Grammatik im Gespräch. Konstruktionen der Selbst- und Fremdpositionierung. Berlin u. a.: De Gruyter. S. 21-56. (= LinguistikImpulse \& Tendenzen 33).

Deppermann, Arnulf/Elstermann, Mechthild (2008): Lexikalische Bedeutung oder Konstruktionsbedeutungen. Eine Untersuchung am Beispiel von Konstruktionen mit ,verstehen'. In: Fischer, Kerstin/Stefanowitsch, Anatol (Hg.): Konstruktionsgrammatik II: Von der Konstruktion zur Grammatik. Tübingen: Stauffenburg. S. 103-134.

Deppermann, Arnulf/Helmer, Henrike (2013): Zur Grammatik des Verstehens im Gespräch: Inferenzen anzeigen und Handlungskonsequenzen ziehen mit ,also' und ,dann'. In: Zeitschrift für Sprachwissenschaft 32, 1. S. 1-39.

Diewald, Gabriele (2006): Discourse particles and modal particles as grammatical elements. In: Fischer, Kerstin (Hg.): Approaches to discourse particles. Amsterdam: Elsevier. S. 403-426. (= Studies in Pragmatics 1).

Diewald, Gabriele (2013): "Same same but different" - Modal particle, discourse markers and the art (and purpose) of categorization. In: Degand, Liesbeth/Cornillie, Bert/Pietrandrea, Paola (Hg.): Discourse markers and modal particles. Categorization and description. Amsterdam u. a.: Benjamins. S. 19-46. (= Pragmatics \& Beyond New Series 234).

Diewald, Gabriele/Fischer, Kerstin (1998): Zur diskursiven und modalen Funktion der Partikeln aber, auch, doch und ja in Instruktionsdialogen. In: Linguistica 38(1). S. 75-99.

Diewald, Gabriele/Kresić, Marijana (2010): Ein übereinzelsprachliches kontrastives Beschreibungsmodell für Partikelbedeutungen. In: Linguistik online 44, 4. S. 5-18.

Dittmar, Norbert (1997): Grundlagen der Soziolinguistik: Ein Arbeitsbuch mit Aufgaben. Tübingen: Niemeyer. (= Konzepte der Sprach- und Literaturwissenschaft 57).

Dittmar, Norbert (2000): Sozialer Umbruch und Sprachwandel am Beispiel der Modalpartikeln halt und eben in der Berliner Kommunikationsgemeinschaft nach der „Wende“. In: Auer, Peter/Hausendorf, Heiko (Hg.): Kommunikation in gesellschaftlichen Umbruchsituationen. Mikroanalytische Aspekte des sprachlichen und gesellschaftlichen Wandels in den Neuen Bundesländern. Tübingen: Niemeyer. S. 199234. (= Reihe Germanistische Linguistik 219).

Ehlich, Konrad (1983): Alltägliches Erzählen. In: Sanders, Willy/Wegenast, Klaus (Hg.): Erzählen für Kinder - Erzählen von Gott. Begegnung zwischen Sprachwissenschaft und Theologie. Stuttgart: Kohlhammer. S. $128-150$.

Eichhoff, Jürgen (1978): Wortatlas der deutschen Umgangssprache. Bern: De Gruyter.

Eisenberg, Peter (1999): Grundriß der deutschen Grammatik. Der Satz. Stuttgart: Metzler.

Fischer, Kerstin (2007): Grounding and common ground: modal particles and their translation equivalents. In: Fetzer, Anita/Fischer, Kerstin (Hg.): Lexical markers of common grounds. Amsterdam: Elsevier. S. 47-66. (= Studies in Pragmatics 3).

Gaines, Philip (2011): The multifunctionality of discourse operator 'okay': Evidence from a police interview. In: Journal of Pragmatics 43. S. 3291-3315.

Goffman, Erving (1967): On Face-Work. In: Goffman, Erving (Hg.): Interaction ritual. Essays on face-toface behavior. New York: Anchor Books. S. 5-45.

Gumperz, John (1992): Contextualization revisited. In: Auer, Peter/Di Luzio, Aldo (Hg.): The contextualization of language. Amsterdam u. a.: Benjamins. S. 39-54. (= Pragmatics \& Beyond New Series 22).

Günthner, Susanne (2015): Zwar...aber-Konstruktionen im gesprochenen Deutsch: Die dialogische Realisierung komplexer Konnektoren im Gespräch. In: Deutsche Sprache 43. S. 193-219. 
Hagemann, Jörg (2009): Tag questions als Evidenzmarker. Formulierungsdynamik, sequentielle Struktur und Funktionen redezuginterner tags. In: Gesprächsforschung - Online-Zeitschrift zur verbalen Interaktion 10. S. 145-176.

Helbig, Gerhard (1988): Lexikon deutscher Partikeln. Leipzig: Verlag Enzyklopädie.

Helbig, Gerhard (1999): Deutsche Grammatik. Grundfragen und Abriß. München: Iudicium.

Helbig, Gerhard/Buscha, Joachim (2001): Deutsche Grammatik. Berlin: Langenscheidt.

Helbig, Gerhard/Helbig, Agnes (1995): Deutsche Partikeln - richtig gebraucht? Leipzig: Verlag Enzyklopädie.

Hentschel, Elke (1986): Funktion und Geschichte deutscher Partikeln. Ja, doch, halt und eben. Tübingen: Niemeyer. (= Reihe Germanistische Linguistik 63).

Hentschel, Elke/Weydt, Harald (2003): Handbuch der deutschen Grammatik. Berlin: De Gruyter.

Ickler, Theodor (1994): Zur Bedeutung der sogenannten ,Modalpartikeln“. In: Sprachwissenschaft 19, 3/4. S. 374-404.

Imo, Wolfgang (2008): Individuelle Konstrukte oder Vorboten einer neuen Konstruktion? Stellungsvarianten der Modalpartikel halt im Vor- und Nachfeld. In: Stefanowitsch, Anatol/Fischer, Kerstin (Hg.): Konstruktionsgrammatik II. Von der Konstruktion zur Grammatik. Tübingen: Stauffenburg. S. 135-156.

Imo, Wolfgang (2016): Grammatik. Eine Einführung. Stuttgart: Metzler.

Kallmeyer, Werner (1987): Bemerkungen zum Verhältnis von Sprache und Interaktion am Beispiel von ,Ja aber‘. In: Russ, Charles/Volkmar, Claudia (Hg.): Sprache und Gesellschaft in deutschsprachigen Ländern. Beiträge eines Werkstattgesprächs des Goethe-Instituts York in Zusammenarbeit mit dem Department of Language and Linguistic Science der Universität York am 16./17.9.1985 in York. München: Goethe-Institut. S. 53-74.

Kwon, Min-Jae (2005): Modalpartikeln und Satzmodus. Untersuchungen zur Syntax, Semantik und Pragmatik der deutschen Modalpartikeln. Dissertation. LMU München.

Labov, William (1972): The transformation of experience in narrative syntax. In: Labov, William (Hg.): Language in the inner city. Studies in the Black English vernacular. Philadelphia: University of Pennsylvania Press. S. 354-396.

Lambrecht, Knud (1994): Information structure and sentence form. Topic, focus, and the mental representations of discourse referents. Cambridge: Cambridge University Press. (= Cambridge Studies in Linguistics 71).

Meer, Dorothee (1998): Der Prüfer ist nicht der König. Mündliche Abschlußprüfungen in der Hochschule. Berlin: De Gruyter. (= Reihe Germanistische Linguistik 202).

Müller, Sonja (2016): Halt eben vs. eben halt - Dialekt, Satzmodus, Rhythmus oder Interpretation? In: Sprachwissenschaft 41, 2. S. 139-184.

Müller, Sonja (2018): Distribution und Interpretation von Modalpartikel-Kombinationen. Berlin: Language Science Press. (= Topics at the Grammar-Discourse Interface 2).

Proske, Nadine (2013a): Die pragmatische Funktion syntaktischer Funktionen in spontan gesprochener Sprache. In: Hagemann, Jörg/Klein, Wolf Peter/Staffeldt, Sven (Hg.): Pragmatischer Standard. Tübingen: Stauffenburg. S. 207-222.

Proske, Nadine (2013b): Informationsmanagement im gesprochenen Deutsch. Eine diskurspragmatische Untersuchung syntaktischer Strukturen in Alltagsgesprächen. Heidelberg: Winter. (= OraLingua 5).

Quasthoff, Uta (2001): Erzählen als interaktive Gesprächsstruktur. In: Brinker, Klaus/Antos, Gerd/Heinemann, Wolfgang/Sager, Sven F. (Hg.): Text- und Gesprächslinguistik. Ein internationales Handbuch zeitgenössischer Forschung. 2. Halbband. Berlin u.a.: De Gruyter. S. 1293-1309. (= Handbücher zur Sprach- und Kommunikationswissenschaft 16.2).

Reineke, Silke (2016): Wissenszuschreibungen in der Interaktion. Eine gesprächsanalytische Untersuchung impliziter und expliziter Formen der Zuschreibung von Wissen. Heidelberg: Winter. (= OraLingua 12). 
Rost-Roth, Martina (1998): Modalpartikeln in Argumentsituationen und Handlungsvorschlägen. In: Harden, Theo/Hentschel, Elke (Hg.): Particulae particularum. Festschrift zum 60. Geburtstag von Harald Weydt. Tübingen: Stauffenburg. S. 293-324.

Sacks, Harvey (1995): Lectures on Conversation. Volumes I \& II. Edited by Gail Jefferson. Oxford: Blackwell.

Schegloff, Emanuel (2007): Sequence Organization in Interaction. A Primer in Conversation Analysis I. Cambridge: Cambridge University Press.

Schlobinski, Peter (1992): Funktionale Grammatik und Sprachbeschreibung. Eine Untersuchung zum gesprochenen Deutsch sowie zum Chinesischen. Opladen: Westdeutscher Verlag.

Schoonjans, Steven (2013): Modal particles: Problems in defining a category. In: Degand, Liesbeth/Cornillie, Bert/Pietrandrea, Paola (Hg.): Discourse markers and modal particles. Categorization and description. Amsterdam u. a.: Benjamins. S. 133-161. (= Pragmatics \& Beyond New Series 234).

Selting, Margret/Auer, Peter/Barth-Weingarten, Dagmar/Bergmann, Jörg/Bergmann, Pia/Birkner, Karin/ Couper-Kuhlen, Elizabeth/Deppermann, Arnulf/Gilles, Peter/Günthner, Susanne/Hartung, Martin/Kern, Friederike (2009): Gesprächsanalytisches Transkriptionssystem 2 (GAT2). In: Gesprächsforschung Online-Zeitschrift zur verbalen Interaktion 10. S. 353-402.

Selting, Margret/Couper-Kuhlen, Elizabeth (2000): Argumente für die Entwicklung einer ,interaktionalen Linguistik'. In: Gesprächsforschung - Online-Zeitschrift zur verbalen Interaktion 1. S. 76-95.

Spranz-Fogasy, Thomas (2005): Argumentation als alltagsweltliche Kommunikationsideologie. In: Deutsche Sprache 33. S. 141-156.

Spreckels, Janet (2009): ,ich hab einfach gedacht': Stellungnahme und Positionierung durch ,einfach ' in Erklärinteraktionen. In: Günthner, Susanne/Bücker, Jörg (Hg.): Grammatik im Gespräch. Konstruktionen der Selbst- und Fremdpositionierung. Berlin u. a.: De Gruyter. S. 115-148. (= Linguistik - Impulse \& Tendenzen 33).

Thurmair, Maria (1989): Modalpartikeln und ihre Kombinationen. Tübingen: Niemeyer. (= Linguistische Arbeiten 223).

Thurmair, Maria (1991): ,Kombinieren Sie doch nur ruhig auch mal Modalpartikeln!‘: Combinatorial regularities for modal particles and their use as an instrument of analysis. In: Multilingua 10, 1/2. S. 19-42.

Watson, Rod (1997): Some General Reflections on 'Categorization' and 'Sequence' in the Analysis of Conversation. In: Hester, Stephen/Eglin, Peter (Hg.): Culture in action. Studies in membership categorization analysis. Washington, D.C.: University Press of America. S. 49-76. (= Studies in Ethnomethodology and Conversation Analysis 4).

Weydt, Harald (1969): Abtönungspartikeln. Die deutschen Modalwörter und ihre französischen Entsprechungen. Bad Homburg: Gehlen. (= linguistica \& litteraria 4).

Zifonun, Gisela/Hoffmann, Ludger/Strecker, Bruno (1997): Grammatik der deutschen Sprache. Berlin u. a.: De Gruyter. (= Schriften des Instituts für Deutsche Sprache 7).

Sarah Torres Cajo, M.A.

Leibniz-Institut für Deutsche Sprache

R5 6-13

68161 Mannheim

E-Mail: torres@ids-mannheim.de 